\title{
On-line Mode Decomposition of Fluid Flows Using Moving Horizon Estimation
}

\author{
A. Alessandri, P. Bagnerini, C. Carmeli, M. Gaggero, D. Lengani, D. Simoni
}

\begin{abstract}
The on-line analysis and monitoring of a turbulent flow across a channel is really important in a number of applications. Unfortunately, such a problem is difficult to address since the flow is governed by the Navier-Stokes equation. Dynamic mode decomposition is usually adopted to analyze such flows via the on-line identification of local linear approximations of spatio-temporal dynamics of the flow velocities, i.e., the square matrix of a linear system. We propose a new approach to mode decomposition based on moving horizon estimation by providing a rigorous proof of stability for the estimation error. Moreover, we address the problem of computing the distance of a given estimated matrix to stability or instability. Such information is important to measure the "degree" of stability/instability for the purpose of control. Numerical results obtained with an experimental dataset are presented and discussed.
\end{abstract}

\section{INTRODUCTION}

In spite of the amount of research reported in the literature, nowadays modeling of turbulent flows is still difficult since flows are described by the Navier-Stokes equation, which is a nonlinear, partial differential equation that has always attracted a lot of investigations from both theoretical and practical point of view but is still difficult to deal. Thus, techniques such as dynamic mode decomposition (DMD) have been proposed to analyze such flows. In this paper, we propose a new approach to mode decomposition based on moving horizon estimation (MHE), which is really wellsuited to being applied in this context for its intrinsic robustness. In order to quickly detect transitions of boundary layers in flows, we will address the problem of measuring the distance of a given unstable matrix to stability and its reverse, i.e., the distance of a stable matrix to instability. Efficient methods based on linear matrix inequalities (LMIs) will be presented to perform such tasks by using semidefinite programming (SDP) [1].

Transition process of boundary layers is a complex phenomenon that is pretty well studied in fluid dynamics [2]-[8]. Low-order models are adopted to reduce the computational burden due to the large amount of measurements to deal with. Such models enable to represent complex "structures" such as vortices in the spatial and temporal distributions (see, e.g., [9]-[11]). Toward this end, DMD is often combined with orthogonal decomposition (POD), as pointed out in [12].

A. Alessandri P. Bagnerini, C. Carmeli, D. Lengani, and D. Simoni are with the University of Genoa, DIME, Genoa, Italy (e-mail: alessandri@dime.unige.it, bagnerini@dime.unige.it, carmeli@dime.unige.it, davide.lengani@edu.unige.it, daniele.simoni@unige.it).

M. Gaggero is with the National Research Council of Italy, Genoa, Italy (e-mail: mauro.gaggero@cnr.it).
DMD allows to identify the main dynamics of a system with direct measurements of the state variables by providing a simpler representation of the response modes and their evolution over time [13]. Using DMD, the dominant dynamics is extracted through the linear best-fitting mapping of successive ensembles of snapshots, and hence it enables to detect instability waves without solving the Navier-Stokes equation [14]. The information raised by DMD regards the estimation of modes as well as their frequency and growth (or decay) rates [13]-[15]. The quality of the results provided by such techniques depends on the complexity of the dynamics of the fluid flow, which is time-varying and strongly affected by external disturbances.

In this paper, we deal with the combination of mode decomposition and MHE to estimate the approximate linear dynamics on line. As compared with the literature on MHE, the results presented here concern the extension of classical results on MHE for linear systems [16], [17] to systems having a square matrix as state. MHE has been successfully applied to estimate the state of nonlinear systems [18]-[20]. Extensions of MHE to estimate the state of switching systems are reported in [21], [22]. Uncertainties have been explicitly considered in several works such as [23]-[26]. Fast MHE based on imperfect optimization of descent algorithms [27] enables to reduce the computational demand and hence deal with a large amount of data in real time. The moving-horizon strategy turns out to be robust to uncertainties due to forcing incorrect modeling (linear instead of nonlinear). For such reasons, the combination of MHE and mode decomposition turns out to be successful, as it will be shown with the results obtained by processing an experimental dataset of velocity flow measurements.

The problem of measuring the distance of a given matrix to stability/instability is pretty well-known (see, for instance, [28] and the reference therein). Most of the results concern continuous-time systems [29]-[31]. Only recently the problem has been addressed for discrete-time systems [32]. Concerning this topic, the main contribution of this work consists in the formulation of LMI-based conditions for the evaluation of such distances. Using LMIs and SDP tools, the computation turns out be easily tractable, which is particularly important when large matrices have to be processed as in our case study.

The paper is organized as follows. The proposed approach is presented in Section II, which includes a stability analysis of the estimation error too. Section III concerns the LMIbased methods we have developed to evaluate the distance between an estimated mode-decomposition matrix and the 
space of stable and unstable matrices. Numerical results obtained with an experimental dataset are reported in Section IV. Section V deals with conclusions and prospects of future work.

We will adopt the following notation. The minimum and maximum eigenvalues of a real, symmetric matrix $P$ are denoted by $\lambda_{\min }(P)$ and $\lambda_{\max }(P)$, respectively. Moreover, $P>0(P \geq 0)$ means that $P$ is positive definite (semidefinite). Given a real matrix $M$, the spectral norm of $M$ is $|M|_{2}:=\left(\lambda_{\max }\left(M^{\top} M\right)\right)^{1 / 2}=\left(\lambda_{\max }\left(M M^{\top}\right)\right)^{1 / 2}$. The Frobenius norm of a real matrix $M$ is $|M|_{F}:=\sqrt{\operatorname{tr}\left(M^{\top} M\right)}$. Given a complex matrix $C, C^{*}$ denotes its Hermitian transpose. Finally, $I$ denotes the identity matrix of appropriate dimension.

\section{Mode Decomposition BASEd On MHE}

DMD enables to fit a series of velocity measurements with the temporal or spatial evolution of a fluid flow by providing the best linear transformation in the the sense of least squares. In practice, we get the "best" linear state equation that accounts for a flow field snapshot sequence into the successive one. The snapshots can be collected over space or time.

Following [13], let us denote by $V_{k}$ the collection of $N$ snapshots from $k-N+1$ to $k$, i.e.,

$$
V_{k}:=\operatorname{col}\left(v_{k-N+1}, v_{k-N+2}, \ldots, v_{k}\right)
$$

where $v_{i} \in \mathbb{R}^{n}, i=1,2, \ldots$, represents a single snapshot. The DMD fitting results from the solution of the minimization problem

$$
S_{k}=\underset{S \in \mathcal{S}}{\operatorname{argmin}}\left|V_{k+1}-V_{k} S\right|_{F}^{2}
$$

where the unknown $S$ is a transformation matrix of appropriate dimension, and in canonical form it is given by

$$
S=\left(\begin{array}{ccccc}
0 & 0 & \cdots & \cdots & a_{1} \\
1 & 0 & \cdots & \cdots & a_{2} \\
& \ddots & \ddots & & \vdots \\
& & 1 & 0 & a_{N-1} \\
& & 0 & 1 & a_{N}
\end{array}\right), a \in \mathbb{R}^{N}
$$

and $\mathcal{S}$ denotes the set of all real matrices in companion form with $N \geq 2$. Thus, DMD is based on Algorithm 1 .

Algorithm 1 has been successfully employed to detect flow transition [33]. In this paper, instead of (1) we will propose a new decomposition method based on MHE by considering the cost function

$$
J_{k}(S)=\mu\left|S-\bar{S}_{k}\right|_{F}^{2}+\left|V_{k+1}-V_{k} S\right|_{F}^{2}
$$

where $\mu \geq 0$ and $\bar{S}_{k} \in \mathbb{R}^{N \times N}$ is a given prediction. A suitable choice of $\bar{S}_{k}$ is just that of $S_{k}$ obtained at the previous step. The parameter $\mu$ enables to weight our trust in the outputs w.r.t. the prediction, i.e., we may select a "small" $\mu$ with a little uncertainty on the measurements. By contrast,
Algorithm 1: given $V_{k}$, apply

1) compute the singular value decomposition of $V_{k}$, i.e., two unitary matrices $U$ and $W$ and a diagonal matrix $\Sigma$ s.t. $V_{k}=U \Sigma W^{*}$

2) compute the projection of the matrix $S_{k}$ on the POD modes given by $M_{k}=U^{*} V_{2} W \Sigma^{-1}$

3) construct the matrices of the eigenvectors and eigenvalues of the matrix $M_{k}$, i.e., $X$ and $D$ (diagonal)

4) construct the matrix of the DMD modes $\Theta=U X$

5) construct the diagonal matrix $Z_{k}$ of the logarithms of the DMD eigenvalues of $M_{k}$, i.e., $Z_{k}:=\log (D) /(2 \pi \Delta t)$, where $\Delta t$ is the time between two consecutive snapshots

and get $Z_{k}$.

we have to increase $\mu$ in case of outputs corrupted by a lot of noise. It is easy to verify that the solution of

$$
\hat{S}_{k}=\underset{S \in \mathbb{R}^{N \times N}}{\operatorname{argmin}} J_{k}(S)
$$

is given by

$$
\hat{S}_{k}=\left(\mu I+V_{k}^{\top} V_{k}\right)^{-1}\left(\mu \bar{S}_{k}+V_{k}^{\top} V_{k+1}\right)
$$

where $k=N, N+1, \ldots$ with $\bar{S}_{k}=\hat{S}_{k-1}$ and some initial "a priori" $\bar{S}_{N}$.

To prove stability, let us assume the following.

Assumption 1: There exists $S^{\circ} \in \mathbb{R}^{N \times N}$ such that

$$
V_{k+1}=V_{k} S^{\circ}+W_{k}, k=N, N+1, \ldots
$$

with $\left|V_{k}\right|_{F} \leq v_{\max }$ and $\left|W_{k}\right|_{F} \leq w_{\max }$ for some $V_{N} \in$ $\mathbb{R}^{n \times N}, v_{\max }, w_{\max }>0$.

The solution given by (4) provides an exponentially bounded estimation error $e_{k}:=S^{\circ}-\hat{S}_{k} \in \mathbb{R}^{N \times N}$, i.e., there exist $\alpha \in(0,1)$ and $\beta>0$ such that $\left|e_{k}\right|_{F} \leq\left|e_{N}\right|_{F} \alpha^{k-N}+$ $\beta, k=N+1, N+2, \ldots$. More specifically, we can state the following.

Theorem 1: Let Assumption 1 hold

$$
\delta:=\inf _{k \geq N+1} \lambda_{\min }\left(V_{k}^{\top} V_{k}\right)
$$

be strictly positive. If

$$
\frac{\mu}{\mu+\delta}<\frac{1}{\sqrt{N}}
$$

the estimation error is exponentially bounded with

$$
\alpha:=\frac{\mu \sqrt{N}}{\mu+\delta} \quad \beta:=\frac{v_{\max } w_{\max } \sqrt{N}}{(1-\sqrt{N}) \mu+\delta} .
$$

Proof. Since $\bar{S}_{k}=\hat{S}_{k-1}$, from (4), it is straightforward to get

$$
\begin{aligned}
\hat{S}_{k}-S^{\circ} & =\mu\left(\mu I+V_{k}^{\top} V_{k}\right)^{-1}\left(\hat{S}_{k-1}-S^{\circ}\right) \\
& +\left(\mu I+V_{k}^{\top} V_{k}\right)^{-1} V_{k} W_{k} .
\end{aligned}
$$

Since

$$
\left(\mu I+V_{k}^{\top} V_{k}\right)^{-1} \leq \frac{I}{\mu+\delta},
$$


from (7) it follows that

$$
\left|\left(\mu I+V_{k}^{\top} V_{k}\right)^{-1}\right|_{2} \leq \frac{1}{\mu+\delta} .
$$

Using the bound $|\cdot|_{F} \leq \sqrt{N}|\cdot|_{2}$, we obtain

$$
\left|e_{k}\right|_{F} \leq \frac{\mu \sqrt{N}}{\mu+\delta}\left|e_{k-1}\right|_{F}+\frac{v_{\max } w_{\max } \sqrt{N}}{\mu+\delta}
$$

and hence

$$
\begin{aligned}
\left|e_{k}\right| & \leq\left(\frac{\mu \sqrt{N}}{\mu+\delta}\right)^{k-N}\left|e_{N}\right|+\frac{v_{\max } w_{\max } \sqrt{N}}{\mu+\delta} \sum_{i=0}^{k-N-1}\left(\frac{\mu \sqrt{N}}{\mu+\delta}\right)^{i} \\
& \leq\left(\frac{\mu \sqrt{N}}{\mu+\delta}\right)^{k-N}\left|e_{N}\right|+\frac{v_{\max } w_{\max } \sqrt{N}}{(1-\sqrt{N}) \mu+\delta}
\end{aligned}
$$

for $k=N+1, N+2, \ldots$, which allows to conclude.

Remark 1: The condition $\delta>0$ requires matrices $V_{k}$ of full rank since otherwise the r.h.s. of (5) turns out to be zero. Condition (6) can be guaranteed by choosing a sufficiently small $\mu$. Generally speaking, the choice $\mu=0$ would correspond to the best transient behavior and asymptotic bound but in practice provides poorly robust estimates since such a bound is quite conservative. Indeed, the choice of $\mu$ should be traded between a fast transient given by a "small" $\mu$ and a robust steady-state behavior with a "large" $\mu$ compatible with (6), i.e., $\mu<\delta /(\sqrt{N}-1)$.

Summarizing, we will rely on the computational procedure given by Algorithm 2, where we combine MHE and POD projection.

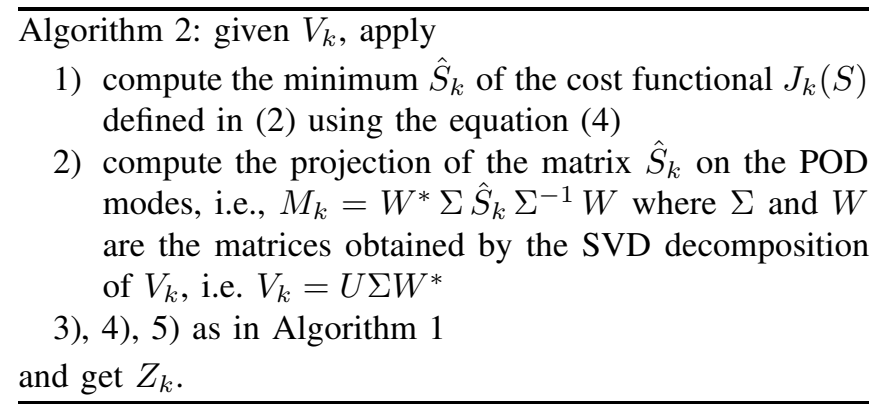

Since we aim at identifying the transition from unstable to stable regime or vice versa, a further goal may be that of minimizing (2) under stability or instability constraints on the minimizer, i.e., for matrices $S \in \mathbb{R}^{N \times N}$ that are (Schur) stable or unstable. Unfortunately, the solution of these problems is difficult but we may get information about the "degree" of stability and instability by computing the distance between a given unstable matrix and the subset of the matrices that are stable or its reverse. This is the topic of the next section.

\section{MATRIX DiSTANCE}

Concerning the methods proposed to compute matrix distance to stability/ instability, the reader is refereed to [34], [35]. In [34] a method to project a given unstable matrix on a convex subset of the space of the stable matrices is proposed. This approach is motivated by the fact that the space of the stable matrices is nonconvex. The problem to find the largest value of instability measures over the admissible uncertainties is addressed in [35], where a sufficient condition for establishing upper bounds on the considered measures is presented and an LMI-based procedure is given to compute such bounds.

\section{A. Distance to stability}

Given any unstable matrix $A \in \mathbb{R}^{m \times m}$, we may evaluate the distance to stability by finding a "small" $E \in \mathbb{R}^{m \times m}$ such that $A+E$ is Schur. Using a formulation based on Lyapunov inequalities, the problem can be rewritten as follows

$$
\begin{aligned}
& \min |E| \quad \text { w.r.t. } \quad E, P \in \mathbb{R}^{m \times m} \quad \text { s.t. } \\
& (A+E)^{\top} P(A+E)-P<0, P>0
\end{aligned}
$$

where $|\cdot|$ is any submultiplicative norm in $\mathbb{R}^{m \times m}$. Using homogeneity and some pretty well-known LMI technicality, (8) can be equivalently formulated as follows

$$
\begin{gathered}
\min |Y| \text { w.r.t. } Y, P \in \mathbb{R}^{m \times m} \\
\left(\begin{array}{cc}
A^{\top} P A+A^{\top} Y+Y^{\top} A-P & \text { s.t. } \\
Y^{\top} & -P
\end{array}\right)<0, P>I
\end{gathered}
$$

with $Y=P E$ since, owing to the condition $P>I$, it follows

$$
|E| \leq\left|P^{-1} Y\right| \leq\left|P^{-1}\right||Y| \leq|Y|
$$

and hence the minimization of $|Y|$ induces to minimize $|E|$. The solution is given by $E=P^{-1} Y$, which is to be regarded as the "smallest" stabilizing matrix for $A$. It is worth noting that (9) can be solved by standard optimization tools based on SDP and hence to deal with large matrices efficiently.

\section{B. Distance to instability}

Let us assume that the matrix $A \in \mathbb{R}^{m \times m}$ is Schur. In [36], the evaluation of the "nearest" unstable matrix has been addressed through the determination of the "smallest" destabilizing matrix for $A$. A matrix $E \in \mathbb{R}^{m \times m}$ is destabilizing for $A$, if $A+E$ is not Schur. Since, by the simple observation in [36],

$$
\sigma_{\min }(A-\lambda I)=\min _{E}\left\{|E|_{F}: \operatorname{det}(A+E-\lambda I)=0\right\}
$$

where $\sigma_{\min }(A-\lambda I)$ is the smallest singular value of $A-\lambda I$, it is clear that we are interested in the determination of

$$
\beta(A):=\min _{|\lambda| \geq 1} \sigma_{\min }(A-\lambda I) .
$$

In general the evaluation of $\beta(A)$ is not easy, and one can try to bound this quantity from above by looking at destabilizing matrices that satisfy the sufficient condition for instability:

$$
(A+E)^{\top} P(A+E)-P \geq 0 .
$$

Such a condition implies that the quadratic Lyapunov function is nondecreasing but it is still difficult to be treated. However, we may rely on a stronger condition that can be solved by using LMIs. Specifically, since $E^{\top} P E \geq 0$ and

$$
(A+E)^{\top} P(A+E)-P=A^{\top} P A+A^{\top} P E+E^{\top} P A
$$




$$
+E^{\top} P E-P
$$

it follows that

$$
A^{\top} P A+A^{\top} P E+E^{\top} P A-P \geq 0
$$

ensures that (10) holds. To reduce the conservativeness of (11), we can exploit the bound

$$
E^{\top} P E \geq \lambda_{\min }\left(E^{\top} P E\right) I
$$

by using Algorithm 3, where, likewise in Section III-A, we get the minimization of $|E|$ by minimizing $|Y|$ owing to the additional constraint $P>I$ and with a sufficiently small $\Delta \lambda>0$.
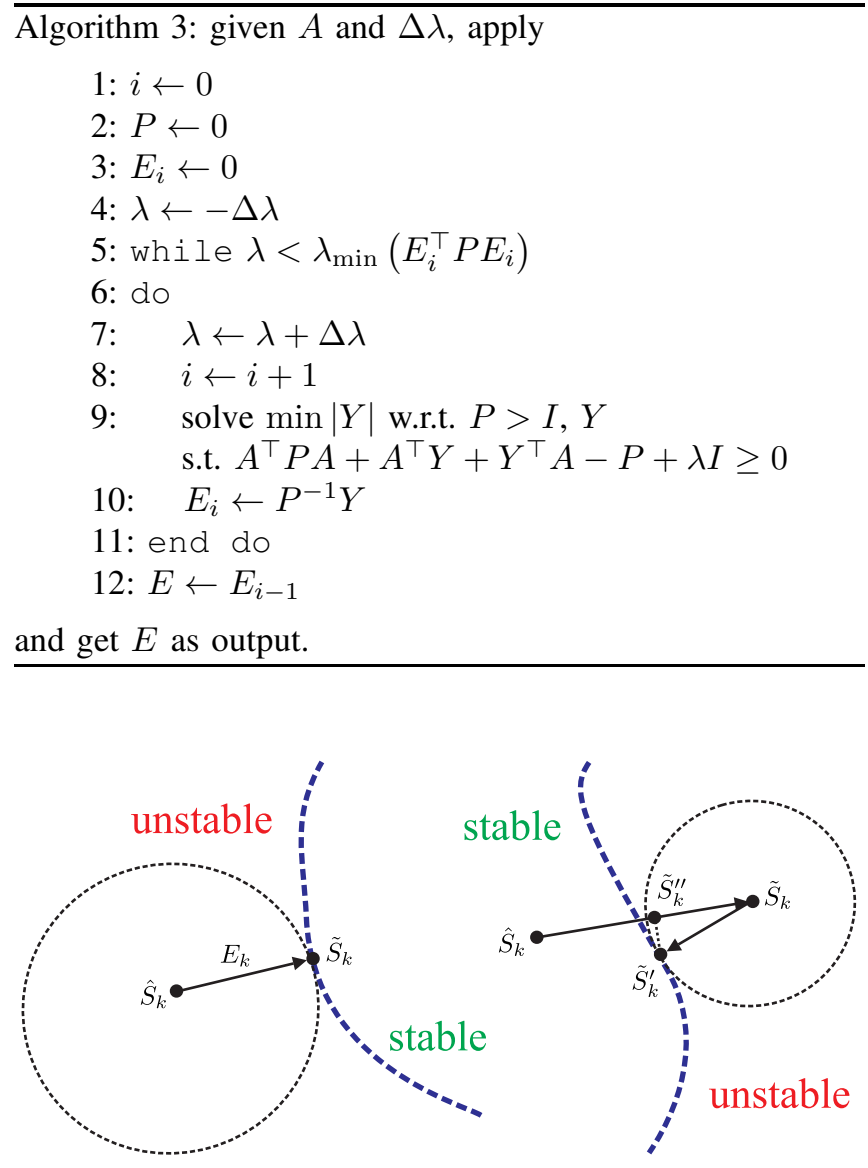

Fig. 1. Illustrations of the application of the proposed methods for matrix distance evaluation, where $E_{k}$ is the solution of (9) with $A=\hat{S}_{k} ; \tilde{S}_{k}^{\prime}$ results from the solution of (9) $A=\tilde{S}_{k} ; \tilde{S}_{k}^{\prime \prime}$ is the projection of $\tilde{S}_{k}^{\prime}$ on the segment with initial/final points given by $\hat{S}_{k}$ and $\tilde{S}_{k}$.

Next section will concern the results obtained with an experimental dataset.

\section{NUMERICAL RESULTS}

The results presented in the following are based on experiments performed in the open-circuit low-speed wind tunnel of the aerodynamics and turbomachinery laboratory of the University of Genoa, Italy. The experimental setup consists of a thick flat plate, where the flat part of the plate includes the leading edge, which is $200 \mathrm{~mm}$ long and

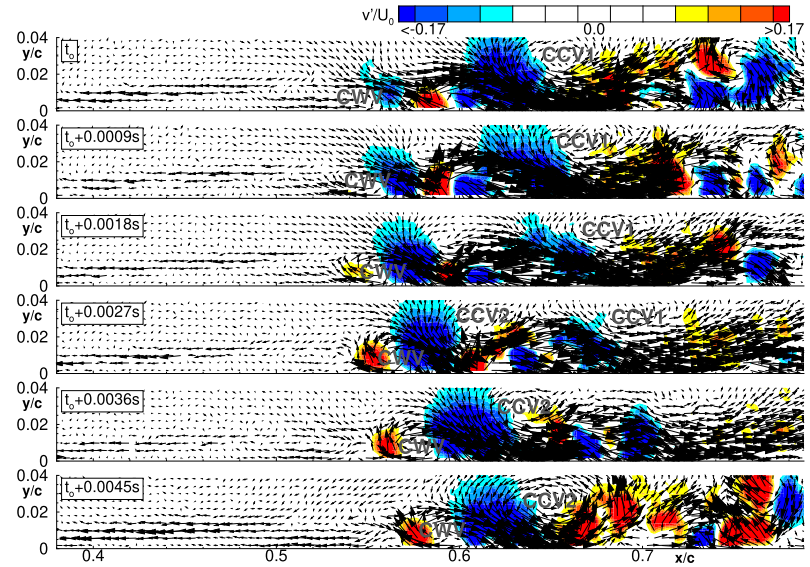

Fig. 2. Sequence of instantaneous perturbation velocity vectors $(\mathrm{Re}$ decomposition), case $R e=75000, F S T I=0.65 \%$.

$300 \mathrm{~mm}$ wide. The plate has been installed between two contoured walls producing an adverse pressure gradient. The boundary layer developing along the rear part of the plate has been surveyed by means of a Dantec time-resolved particle image velocimetry (TR-PIV). The measuring domain extends from $x / c=0.315$ to $x / c=0.9$ and the test section throat is located at $x / c=0.285$, where $c$ denotes the plate length. A dataset of 3100 instantaneous velocity fields has been acquired at a sampling rate of $3100 \mathrm{~Hz}$. The high-frequency resolution and the long sampling period $(1 \mathrm{~s})$ of the dataset allow to follow the dynamics leading to the generation of the large scale coherent structures, and hence to the transition.

The tests have been carried out with subsonic flow condition at a Reynolds number of 75000 (based on the plate length and the inlet flow velocity) and a free-stream turbulence intensity level of $0.65 \%$ (measured at the leading edge of the plate), as shown Fig. 2. In the first instants, a clock-wise rotating vortex denoted by CWV arises. Next, large counter-clockwise rotating vortical structures denoted by CCV1 and CCV2 can be observed as well.

The proposed MHE-based decomposition is applied to this large dataset of measurements provided by the TR-PIV. When both streamwise and wall-normal velocity components is available, it has been shown that the wall-normal velocity component is more representative of the change of regime since before transition starts it is almost null [37] and thus, for the sake of space limitation, we will focus only on such a component. Both components are instead considered to compute frequencies and growth rates, as the whole information is needed to obtain a satisfactory accuracy.

The MHE algorithm described in Section II is applied to the sequence of snapshots from $k=k_{0}$ to $k=k_{f}$ with $k_{0}=40$ and $k_{f}=160$. The length $N$ of the MHE window is chosen equal to 38. At each $k$, the exact solution $\hat{S}_{k}$ minimizing (2) is computed using (4). The initial matrix $\bar{S}_{k_{0}}$ is chosen by performing the classical DMD. Based on the current estimate $\hat{S}_{k}$, we compute the residual norm

$$
r_{k}:=\left|V_{k+1}-V_{k} \hat{S}_{k}\right|_{F}
$$


and, following [13], we project $\hat{S}_{k}$ on the POD modes by obtaining

$$
M_{k}=W^{*} \Sigma \hat{S}_{k} \Sigma^{-1} W
$$

where $\Sigma$ and $W$ are the matrices obtained by the SVD decomposition of $V_{k}$, i.e. $V_{k}=U \Sigma W^{*}$. The matrices of the eigenvectors and eigenvalues of $M_{k}$ are then computed as described in the Algorithm 2. Notice that the resulting matrix $\hat{S}_{k}$ is not companion due to the presence of the first term in (2).

The frequency information is contained in the imaginary part of such eigenvalues, while the real part provides the growth/decay rate of the dynamic structure identified by the corresponding DMD mode. Positive values of the real part of the DMD eigenvalues indicate growing structures, corresponding to unstable waves. Stable regimes such as the pre-transitional region and the fully turbulent one are instead characterized by negative or null growth rates.

The results obtained by applying Algorithm 2 with $\mu=$ 100 and $N=38$ are shown in Figs. 3 and 4. Fig. 3 illustrates the growth rate, corresponding to the maximum real part of the eigenvalues of $M_{k}$ for each value of $k$. It is clearly visible that the growth rate is negative in the stable region as no unstable eigenvalues have been observed. Then, the growth rate increases its value and reaches a maximum in the unstable region where becomes positive, and then decreases again in the turbulent stable regime as expected.

In Fig. 4 the residual norm is depicted. It is possible to notice a decreasing trend in the stable regime since the pre-transitional region is ruled by linear effects and low velocity fluctuations, so that the residual norm decreases when the number of snapshots increases. The decreasing trend is followed by an increasing one in the transitional regime, where the velocity fluctuations increase significantly and hence the residuals grow as well. Finally, the residual norm still decreases in the turbulent regime when linear instability ends and is definitively damped by the nonlinear terms.

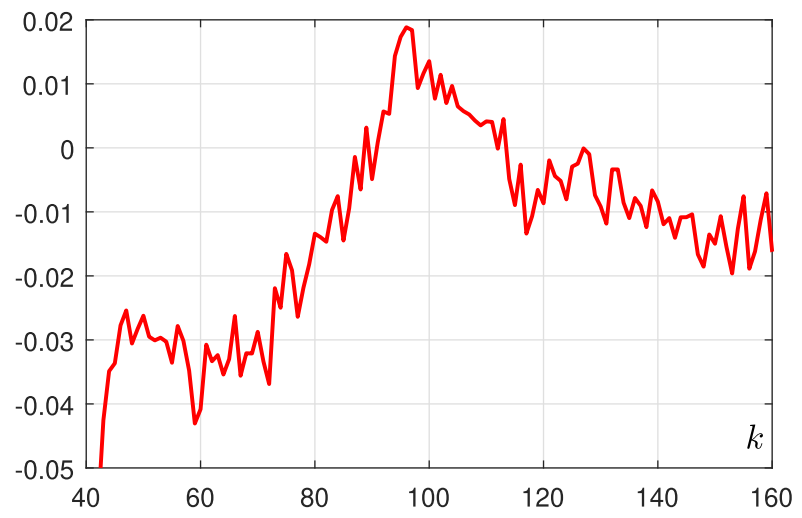

Fig. 3. Growth rate corresponding to the maximum real part of the eigenvalues of $M_{k}$.

According to Section III-A, the matrix $\hat{S}_{k}$ computed at each $k$ is projected on the convex subset of the space

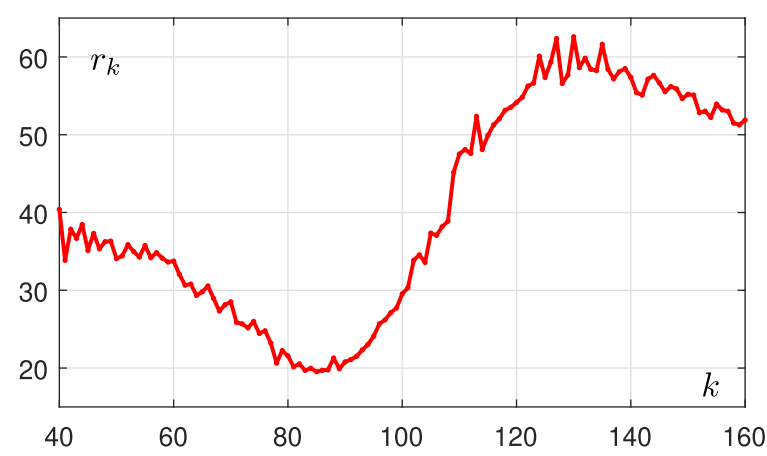

Fig. 4. Residual norm resulting from the application of the MHE algorithm.

of the stable matrices and the corresponding distance is thus evaluated, as shown in Fig. 5. The distance is zero in the linear stable regime and in the turbulent one since the corresponding matrices are stable. In the pre-transitional unstable regime, the value of the distance increases until a maximum (in correspondence of the largest instability) and then decreases until it becomes zero in the turbulent region. Similarly, following Section III-B, the bound on the distance of the matrix $\hat{S}_{k}$ to the set of unstable matrices is computed, as shown in Fig. 6. The distance is large in the first laminar regime and becomes zero in the unstable one. In the turbulent region the distance is different from zero, but smaller with respect to laminar region since in this flow regime reminiscent effects of instability can be still present into the flow, even though they are dominated by nonlinear effects.

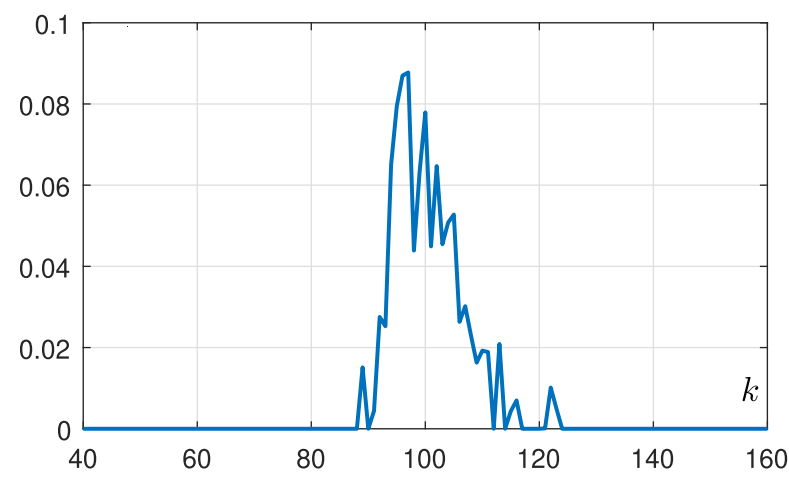

Fig. 5. Distance of the matrix $\hat{S}_{k}$ to the set of the stable matrices with $\mu=100$.

\section{CONCLUSIONS}

The results obtained by applying the proposed MHE-based decomposition to an experimental dataset are quite good. The method to find the distance of an estimated unstable matrix to stability performs quite well too. By contrast, the approach for computing the distance to instability needs to be refined. However, a promising direction.

As a topic of future investigation, we will address the reduction of the computational effort by using fast MHE 


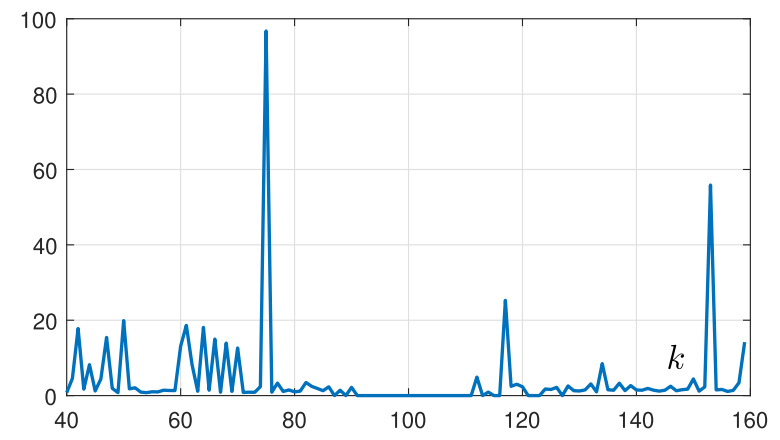

Fig. 6. Upper bound on the distance of the matrix $\hat{S}_{k}$ to the set of the unstable matrices.

techniques [27], which need to be redesigned since the state is given by large square matrices.

\section{REFERENCES}

[1] S. Boyd, L. El Ghaoui, E. Feron, and V. Balakrishnan, Linear Matrix Inequalities in System and Control Theory, ser. Studies in Applied Mathematics. Philadelphia, PA: SIAM, 1994, vol. 15.

[2] T. Zaki, "From streaks to spots and on to turbulence: Exploring the dynamics of boundary layer transition," Flow Turbul. and Combust., vol. 91, pp. 451-473, 2013.

[3] L. Bradt, F. Schlatter, and D. S. Henningson, "Transition in boundary layers subject to free-stream turbulence," Journal of Fluid Mechanics, vol. 517, p. 167-198, 2004.

[4] V. Nagabhushana Rao, R. Jefferson-Loveday, P. G. Tucker, and S. Lardeau, "Large eddy simulations in turbines: Influence of roughness and free-stream turbulence," Flow Turbul. and Combust., vol. 92, pp. 543-561, 2014.

[5] D. Lengani, D. Simoni, M. Ubaldi, P. Zunino, and F. Bertini, "Experimental study of free-stream turbulence induced transition in an adverse pressure gradient," Experimental Thermal and Fluid Science, vol. 84, pp. 18-27, 2017.

[6] O. Marxen, M. Lang, and U. Rist, "Vortex formation and vortex breakup in a laminar separation bubble," Journal of Fluid Mechanics, vol. 728, pp. 58-90, 2013.

[7] T. Michelis, S. Yarusevych, and M. Kotsonis, "On the origin of spanwise vortex deformations in laminar separation bubbles," Journal of Fluid Mechanics, vol. 841, pp. 81-108, 2018.

[8] S. Hosseinverdi and H. F. Fasel, "Numerical investigation of laminarturbulent transition in laminar separation bubbles: the effect of freestream turbulence," Journal of Fluid Mechanics, vol. 858, pp. 714-759, 2019.

[9] C. W. Rowley, T. Colonius, and R. M. Murray, "Model reduction for compressible flows using pod and galerkin projection," Physica D: Nonlinear Phenomena, vol. 189, no. 1-2, pp. 115-129, 2004.

[10] D. Lengani, D. Simoni, M. Ubaldi, P. Zunino, and F. Bertini, "Analysis of the Reynolds stress component production in a laminar separation bubble," International Journal of Heat and Fluid Flow, vol. 64, pp. 112-119, 2017.

[11] D. Lengani, D. Simoni, R. Pichler, R. Sandberg, V. Michelassi, and F. Bertini, "Identification and quantification of losses in a LPT cascade by POD applied to LES data," International Journal of Heat and Fluid Flow, vol. 70, pp. 28-40, 2018.

[12] D. Simoni, D. Lengani, M. Ubaldi, P. Zunino, and M. Dellacasagrande, "Inspection of the dynamic properties of laminar separation bubbles: free-stream turbulence intensity effects for different Reynolds numbers," Experiments in Fluids, vol. 58, no. 6, p. 66, 2017.

[13] P. Schmid, "Dynamic mode decomposition of numerical and experimental data," Journal of Fluid Mechanics, vol. 656, pp. 5-28, 2010.

[14] S. Sarmast, R. Dadfar, R. F. Mikkelsen, P. Schlatter, S. Ivanell, J. Sorensen, and D. S. Henningson, "Mutual inductance instability of the tip vortices behind a wind turbine," J. Fluid Mech., vol. 755, pp. 705-731, 2014.
[15] K. Chen, J. Tu, and C. Rowley, "Variants of dynamic mode decomposition: boundary condition, Koopman, and Fourier analyses," Journal of Nonlinear Science, vol. 22, no. 6, pp. 887-915, 2012.

[16] C. Rao, J. Rawlings, and J. Lee, "Constrained linear estimation-a moving horizon approach," Automatica, vol. 37, no. 10, pp. 16191628,2001

[17] A. Alessandri, M. Baglietto, and G. Battistelli, "Receding-horizon estimation for discrete-time linear systems," IEEE Trans. Automat. Control, vol. 48, no. 3, pp. 473-478, 2003.

[18] C. Rao, J. Rawlings, and D. Mayne, "Constrained state estimation for nonlinear discrete-time systems: stability and moving horizon approximations," IEEE Trans. Automat. Control, vol. 48, no. 2, pp. 246-257, 2003

[19] A. Alessandri, M. Baglietto, and G. Battistelli, "Moving-horizon state estimation for nonlinear discrete-time systems: New stability results and approximation schemes," Automatica, vol. 44, no. 7, pp. 1753 $1765,2008$.

[20] A. Alessandri, M. Baglietto, G. Battistelli, and M. Gaggero, "Movinghorizon state estimation for nonlinear systems using neural networks," IEEE Trans. Neural Networks, vol. 22, no. 5, pp. 768-780, 2011.

[21] A. Alessandri, M. Baglietto, and G. Battistelli, "Receding-horizon estimation for switching discrete-time linear systems," IEEE Trans. Automat. Control, vol. 50, no. 11, pp. 1736-1748, 2005.

[22] Y. Guo and B. Huang, "Moving horizon estimation for switching nonlinear systems," Automatica, vol. 49, no. 11, pp. 3270-3281, 2013.

[23] A. Alessandri, M. Baglietto, and G. Battistelli, "Min-max movinghorizon estimation for uncertain discrete-time systems," SIAM J. Control and Optimization, vol. 50, no. 3, pp. 1439-1465, 2012.

[24] L. Fagiano and C. Novara, "A combined moving horizon and direct virtual sensor approach for constrained nonlinear estimation," Automatica, vol. 49, no. 1, pp. 193-199, 2013.

[25] A. Alessandri and M. Awawdeh, "Moving-horizon estimation with guaranteed robustness for discrete-time linear systems and measurements subject to outliers," Automatica, vol. 67, pp. 85-93, 2016.

[26] Y. Wan, T. Keviczky, M. Verhaegen, and F. Gustafsson, "Data-driven robust receding horizon fault estimation," Automatica, vol. 71, pp. 210-221, 2016.

[27] A. Alessandri and M. Gaggero, "Fast moving horizon state estimation for discrete-time systems using single and multi iteration descent methods," IEEE Trans. Automat. Control, vol. 62, no. 9, pp. 44994511, 2017.

[28] G. H. Golub and C. F. Van Loan, Matrix Computations, 3rd ed. The Johns Hopkins University Press, 1996.

[29] M. Freitag and A. Spence, "A Newton-based method for the calculation of the distance to instability," Linear Algebra and its Applications, vol. 435, no. 12, pp. $3189-3205,2011$.

[30] G. Halikias, N. Karcanias, and A. Papageorgiou, "The distance to strong stability," Linear Algebra and its Applications, vol. 439, no. 10, pp. 2721 - 2735, 2013.

[31] N. Gillis and P. Sharma, "On computing the distance to stability for matrices using linear dissipative Hamiltonian systems," Automatica, vol. 85, pp. $113-121,2017$.

[32] N. Guglielmi and V. Y. Protasov, "On the closest stable/unstable nonnegative matrix and related stability radii," vol. 39 , no. 4, pp. 1642 1669, 2018.

[33] A. Alessandri, P. Bagnerini, M. Gaggero, D. Lengani, and D. Simoni, "Moving horizon trend identification based on switching models for data driven decomposition of fluid flows," in 57th IEEE Conf. on Decision and Control, Miami, Florida, USA, 2018, pp. 2138-2143.

[34] F.-X. Orbandexivry, Y. Nesterov, and P. V. Dooren, "Nearest stable system using successive convex approximations," Automatica, vol. 49, no. 5, pp. $1195-1203,2013$.

[35] G. Chesi, "Instability analysis of uncertain systems via determinants and LMIs," IEEE Trans. Automatic Control, vol. 60, no. 9, pp. 2458$2463,2015$.

[36] C. Van Loan, "How near is a stable matrix to an unstable matrix?" in Linear algebra and its role in systems theory (Brunswick, Maine, 1984), ser. Contemp. Math. Amer. Math. Soc., Providence, RI, 1985, vol. 47, pp. 465-478.

[37] D. Lengani, D. Simoni, M. Ubaldi, P. Zunino, and F. Bertini, "Experimental investigation on the time-space evolution of a laminar separation bubble by proper orthogonal decomposition and dynamic mode decomposition," Journal of Turbomachinery, vol. 139, no. 3, p. 031006, 2017. 